\title{
Neutrino and collider implications of a left-right extended Zee model
}

\author{
Sarif Khan, ${ }^{1,2, *}$ Manimala Mitra, ${ }^{3,2, \dagger}$ and Ayon Patra, ${ }^{4, *}$ \\ ${ }^{1}$ Harish-Chandra Research Institute, Jhunsi, Allahabad 211019, India \\ ${ }^{2}$ Homi Bhabha National Institute, Training School Complex, Anushakti Nagar, Mumbai 400085, India \\ ${ }^{3}$ Institute of Physics, Sachivalaya Marg, Sainik School Post, Bhubaneswar 751005, India \\ ${ }^{4}$ Centre for High Energy Physics, Indian Institute of Science, Bangalore 560012, India
}

(Received 9 June 2018; published 28 December 2018)

\begin{abstract}
We study a simple left-right symmetric extension of the Zee model for neutrino mass generation. An extra $S U(2)_{L / R}$ singlet charged scalar helps in generating a loop-induced Majorana mass for neutrinos in this model. The right-handed neutrinos in this case are very light of the order of a few electron volts to a few mega-electron-volts, which makes this scenario quite different from other left-right symmetric models. We have analyzed the scalar potential and Higgs spectrum in detail, which also play an important role for the neutrino phenomenology. We identified the parameter regions in the model which satisfy the experimentally observed neutrino masses and mixings along with other experimental constraints. We then studied the collider signatures of the charged scalar at $e^{+} e^{-}$colliders with different benchmark points. It is possible to get a huge enhancement in the production cross section of the charged scalar at a lepton collider compared to the hadron colliders, resulting in a much stronger signal, which can be easily observed at the upcoming International Linear Collider or Compact Linear Collider experiments.
\end{abstract}

DOI: 10.1103/PhysRevD.98.115038

\section{INTRODUCTION}

The observation of neutrino oscillation leading to the realization that neutrinos are massive is one of the biggest motivations for physics beyond the Standard Model (SM). A large number of models have been suggested to explain neutrino masses and mixings either by the seesaw mechanism [1] or through loop-induced processes [2]. The Zee model [3] is one of the simplest such scenarios in which neutrino masses are generated at one loop by extending the SM scalar sector with an extra doublet and a charged singlet scalar field. The charged singlet scalar can mix with other charged scalars while also having nonzero flavor-violating couplings with leptons, giving rise to neutrino masses at one loop. Unfortunately the simplest form of the Zee model was shown to be ruled out by experimental neutrino data [4]. However, its extensions might still be viable. In this work, we study an extended Zee model in a left-right symmetric (LRS) framework [5]. The model was proposed and studied in context of the LHC in Ref. [6], and the low-energy

\footnotetext{
*sarifkhan@hri.res.in

†manimala@iopb.res.in

"ayon@okstate.edu
}

Published by the American Physical Society under the terms of the Creative Commons Attribution 4.0 International license. Further distribution of this work must maintain attribution to the author(s) and the published article's title, journal citation, and DOI. Funded by SCOAP . flavor-violating processes were discussed in Ref. [7]. In this work, we examine its viability from neutrino oscillation data, study the scalar potential in detail, and derive charged Higgs spectrum as well as analyze the possible electron-positron collider implications for the charged singlet Higgs boson.

LRS models are attractive extensions of the SM with the gauge group being extended to $S U(3)_{C} \times S U(2)_{L} \times$ $S U(2)_{R} \times U(1)_{B-L}$. The parity symmetry is fundamentally conserved in these models, which provides a natural solution to the strong $C P$ problem [8] without introducing a global Peccei-Quinn symmetry. The parity symmetry is broken once the $S U(2)_{R} \times U(1)_{B-L}$ is spontaneously broken into $U(1)_{Y}$ at a scale $v_{R}$ much above the electroweak scale. Thus, the observed parity violation in the SM can be easily understood. The gauge structure of LRS framework naturally requires the existence of right-handed neutrinos, which can help generate light neutrino masses through the seesaw mechanism. This usually requires the presence of an $S U(2)_{R}$ triplet scalar of which the neutral component acquires a nonzero vacuum expectation value, leading to the right-handed symmetry breaking and the generation of Majorana masses for the right-handed neutrinos. The simplest LRS scenario, on the other hand, requires only an $S U(2)_{R}$ doublet scalar to achieve a consistent right-handed symmetry breaking but cannot generate light neutrino masses. ${ }^{1}$ A simple LRS framework

\footnotetext{
${ }^{1}$ These models need extra singlet fermions for neutrino mass generation.
} 
consisting of two doublets and a bidoublet scalar field, as will be considered here, can only generate a Dirac mass term for the neutrinos, and the introduction of an extra charged singlet scalar is a very economical way to generate neutrino Majorana masses in such a scenario. Hence, it is quite natural to extend the Zee model in a simple LRS framework to generate the neutrino masses and mixings.

There are several other advantages of the LRS extended Zee model. First, since the neutrino Majorana masses are generated at one loop, the right-handed neutrino masses also remain quite light, ranging from a few electron volts to a few mega-electron-volts. This is quite different from other LRS scenarios, in which right-handed neutrinos are very heavy with masses proportional to the right-handed symmetry breaking scale (typically more than a few tera-electron-volts). The presence of lighter right-handed neutrino states is a unique feature of this model. The recent results from the LSND $[9,10]$ and MiniBooNE experiments [11-13] hint at the existence of a light sterile neutrino with mass around a few electron volts. The LRS Zee model would be a prime candidate for explaining such a particle if these experimental results were to persist. Another important consequence of light right-handed neutrinos is the enhanced cross section for the production of the $S U(2)_{L / R}$ singlet charged Higgs boson in this model, especially in the context of electron-positron colliders. The singlet charged Higgs bosons can be pair produced via a $t$-channel process. This process can either be mediated by a left-handed or a right-handed neutrino. The left-handed neutrino mediated processes suffer from extremely small couplings, while the right-handed neutrino mediated processes (for models with heavy right-handed neutrinos) are suppressed by the large right-handed neutrino masses. The $t$-channel mediated charged Higgs pair-production cross section thus remains extremely small for both of these processes. Our scenario, with light mega-electron-volt-scale right-handed neutrinos, can alleviate this shortcoming and deliver a large pairproduction cross section for the charged Higgs boson. Owing to the large couplings with the leptons, the charged singlet Higgs bosons can be copiously produced at lepton colliders and thus give rise to rich collider phenomenology. Since the singlet charged Higgs does not interact with the quarks of the SM, it has a limited discovery prospect in the hadronic colliders including the LHC. A lepton collider, instead, is a perfect setup to test the singlet charged Higgs of this model.

In this work, we pursue a detailed study of the LRS extended Zee model by analyzing the neutrino mass and mixing constraints on the model parameters, taking into account three generations of light neutrinos. We explicitly show the hierarchical structure of the Dirac mass matrix. We also analyze the potential and evaluate the Higgs spectrum in detail. Furthermore, with the set of model parameters that satisfy neutrino oscillation measurements, we carry out an in-depth analysis of the pair production and decay of these charged scalars in the upcoming International Linear Collider (ILC) and Compact Linear Collider (CLIC) experiments. The final state of two opposite-sign leptons and missing energy can be measured quite significantly over the SM background, resulting in a possibility to observe such a process even with a very low luminosity $\mathcal{L} \sim 1-3 \mathrm{fb}^{-1}$ at these experiments. Therefore, even an early run of the ILC/CLIC can detect the presence of such a gauge singlet charged Higgs state.

The rest of this paper is organized as follows. We discuss the model and the particle spectrum in Sec. II. Following that, the pair production of the charged Higgs and its detailed collider phenomenology is discussed in Sec. III. We present our conclusions in Sec. IV.

\section{MODEL AND SPECTRUM}

LRS models are simple gauge extensions of the SM with the gauge group being $S U(3)_{C} \times S U(2)_{L} \times S U(2)_{R} \times$ $U(1)_{B-L}$. The charge of a particle in this model is defined as

$$
\mathcal{Q}=I_{3 L}+I_{3 R}+\frac{B-L}{2},
$$

where $I_{3 L / 3 R}$ is the third component of isospin under $S U(2)_{L / R}$ symmetry. The quarks and leptons consist of three generations of left-handed and right-handed doublet fields,

$$
\begin{array}{rlrl}
Q_{L}\left(3,2,1, \frac{1}{3}\right) & =\left(\begin{array}{l}
u \\
d
\end{array}\right)_{L}, & Q_{R}\left(3,1,2, \frac{1}{3}\right)=\left(\begin{array}{l}
u \\
d
\end{array}\right)_{R}, \\
l_{L}(1,2,1,-1) & =\left(\begin{array}{l}
\nu \\
e
\end{array}\right)_{L}, & l_{R}(1,1,2,-1) & =\left(\begin{array}{l}
\nu \\
e
\end{array}\right)_{R},
\end{array}
$$

where the numbers in the brackets denote the quantum numbers under $S U(3)_{C}, S U(2)_{L}, S U(2)_{R}$, and $U(1)_{B-L}$ gauge groups, respectively. Here, we see that the righthanded neutrinos are naturally present due to the gauge symmetry of the models.

The minimal Higgs sector, required for a consistent symmetry breaking mechanism and the generation of quark and lepton masses and mixing angles, consists of

$$
\begin{gathered}
H_{R}(1,1,2,1)=\left(\begin{array}{c}
H_{R}^{+} \\
H_{R}^{0}
\end{array}\right), \quad H_{L}(1,2,1,1)=\left(\begin{array}{c}
H_{L}^{+} \\
H_{L}^{0}
\end{array}\right), \\
\Phi(1,2,2,0)=\left(\begin{array}{ll}
\phi_{1}^{0} & \phi_{2}^{+} \\
\phi_{1}^{-} & \phi_{2}^{0}
\end{array}\right), \quad \delta(1,1,1,2)=\delta^{+} .
\end{gathered}
$$

The right-handed doublet field $H_{R}$ is required for breaking the $S U(2)_{R} \times U(1)_{B-L}$ into $U(1)_{Y}$ at some high scale to obtain the SM gauge symmetry at the electroweak (EW) 
scale. The $H_{L}$ doublet is required for the preservation of the left-right symmetry. The bidoublet field $\Phi$ is responsible for generation of quark and charged lepton masses and Cabibbo-Kobayashi-Maskawa (CKM) mixing angles. The charged singlet field $\delta^{ \pm}$is needed for the generation of neutrino masses through one-loop diagrams, as will be discussed later in this section.

The Yukawa Lagrangian is given as

$$
\begin{aligned}
\mathcal{L}_{Y}= & Y_{i j}^{q 1} \bar{Q}_{L i} \Phi Q_{R j}+Y_{i j}^{q 2} \bar{Q}_{L i} \tilde{\Phi} Q_{R j}+Y_{i j}^{l 1} \bar{l}_{L i} \Phi l_{R j} \\
& +Y_{i j}^{l 2} \bar{l}_{L i} \tilde{\Phi} l_{R j}+\lambda_{L_{i j}} l_{L i}^{T} i \tau_{2} l_{L j} \delta^{+} \\
& +\lambda_{R_{i j}} l_{R i}^{T} i \tau_{2} l_{R j} \delta^{+}+\text {H.c. }
\end{aligned}
$$

where $Y$ and $\lambda$ are the Yukawa couplings and

$$
\tilde{\Phi}=\tau_{2} \Phi^{*} \tau_{2}
$$

The structure of the $\lambda_{L / R_{i j}}$ term is such that the only terms that will survive are the ones with $i \neq j$. This is exactly the same as in the Zee mechanism of neutrino mass generation. If we expand out any one of the terms involving $\delta^{+}$in the Yukawa Lagrangian, we will get

$$
\mathcal{L} \supset \sum_{i \neq j} \nu_{i} e_{j}\left(\lambda_{i j}-\lambda_{j i}\right),
$$

where $\nu_{i}$ and $e_{j}$ are both in the flavor basis. Thus, if we redefine the $\lambda$ matrix to $\lambda_{i j}^{\prime}=\lambda_{i j}-\lambda_{j i}$, then this new $\lambda^{\prime}$ matrix is completely antisymmetric, and the Lagrangian terms can now be written as

$$
\mathcal{L} \supset \sum_{i, j} \nu_{i} e_{j} \lambda_{i j}^{\prime}
$$

The vacuum expectation values (VEVs) of the Higgs fields are given as $\left\langle\phi_{1}^{0}\right\rangle=v_{1}, \quad\left\langle\phi_{2}^{0}\right\rangle=v_{2}, \quad\left\langle H_{R}^{0}\right\rangle=v_{R}, \quad\left\langle H_{L}^{0}\right\rangle=v_{L}$,

with the effective EW VEV given as $v_{\mathrm{EW}}=\sqrt{v_{1}^{2}+v_{2}^{2}+v_{L}^{2}}$. Without loss of generality, one of the bidoublet VEVs can be chosen to be small. Also, since $v_{L}$ does not contribute to the top mass, a large $v_{L}$ would automatically require a large top Yukawa coupling resulting in the theory being nonperturbative at quite low scales. The hierarchy in the VEVs thus has been chosen such that

$$
v_{R} \gg v_{1}>v_{2}, v_{L} .
$$

The gauge sector of the model consists of two charged $W_{R}^{ \pm}$and $W^{ \pm}$gauge bosons and three neutral bosons including $Z_{R}, Z$, and the photon. The $W_{R}^{ \pm}$and the $Z_{R}$ bosons get their masses at the right-handed symmetry breaking scale and remain heavy, while the others are the same as in the SM. The heavy gauge boson masses in this model are given as

$$
\begin{aligned}
M_{W_{R}^{ \pm}}^{2} & \simeq \frac{1}{2} g_{R}^{2}\left(v_{R}^{2}+v_{1}^{2}+v_{2}^{2}\right), \\
M_{Z_{R}}^{2} & \simeq \frac{1}{2}\left[\left(g_{R}^{2}+g_{V}^{2}\right) v_{R}^{2}+\frac{g_{R}^{4}\left(v_{1}^{2}+v_{2}^{2}\right)+g_{V}^{4} v_{L}^{2}}{g_{R}^{2}+g_{V}^{2}}\right],
\end{aligned}
$$

where $g_{R}$ and $g_{V}$ are the $S U(2)_{R}$ and $U(1)_{B-L}$ gauge couplings, respectively. The left-handed (SM-like) gauge boson masses are given by their usual expressions with the effective $U(1)_{Y}$ gauge coupling $g_{Y}$ given as

$$
g_{Y}=\frac{g_{R} g_{V}}{\sqrt{g_{R}^{2}+g_{V}^{2}}} .
$$

The scalar potential of this model is given as

$$
\begin{aligned}
V(\Delta, \Phi)= & -\mu_{1}^{2} \operatorname{Tr}\left(\Phi^{\dagger} \Phi\right)-\mu_{2}^{2} \operatorname{Tr}\left[\tilde{\Phi} \Phi^{\dagger}+\tilde{\Phi}^{\dagger} \Phi\right]-\mu_{3}^{2} H_{R}^{\dagger} H_{R}-\mu_{4}^{2} H_{L}^{\dagger} H_{L}-\mu_{5}^{2} \delta^{+} \delta^{-}+\left(M_{1} H_{L}^{\dagger} \Phi H_{R}\right. \\
& \left.+M_{2} H_{L}^{\dagger} \tilde{\Phi} H_{R}+\text { H.c. }\right)+\lambda_{1}\left[\operatorname{Tr}\left(\Phi^{\dagger} \Phi\right)\right]^{2}+\lambda_{2}\left[\left\{\operatorname{Tr}\left(\tilde{\Phi} \Phi^{\dagger}\right)\right\}^{2}+\left\{\operatorname{Tr}\left(\tilde{\Phi}^{\dagger} \Phi\right)\right\}^{2}\right]+\lambda_{3} \operatorname{Tr}\left(\tilde{\Phi} \Phi^{\dagger}\right) \operatorname{Tr}\left(\tilde{\Phi}^{\dagger} \Phi\right) \\
& +\lambda_{4} \operatorname{Tr}\left(\Phi \Phi^{\dagger}\right)\left[\operatorname{Tr}\left(\tilde{\Phi} \Phi^{\dagger}\right)+\operatorname{Tr}\left(\tilde{\Phi}^{\dagger} \Phi\right)\right]+\left[i \alpha_{1} \operatorname{Tr}\left(H_{L}^{T} \tau_{2} \Phi H_{R} \delta^{-}\right)+i \alpha_{2} \operatorname{Tr}\left(H_{L}^{T} \tau_{2} \tilde{\Phi} H_{R} \delta^{-}\right)+\text {H.c. }\right] \\
& +\alpha_{3}\left(H_{L}^{\dagger} \Phi \Phi^{\dagger} H_{L}\right)+\alpha_{4}\left[\operatorname{Tr}\left(\tilde{\Phi} \Phi^{\dagger}+\tilde{\Phi}^{\dagger} \Phi\right) H_{L}^{\dagger} H_{L}\right]+\alpha_{5} \operatorname{Tr}\left(\Phi \Phi^{\dagger}\right) H_{L}^{\dagger} H_{L}+\alpha_{6}\left(H_{R}^{\dagger} \Phi^{\dagger} \Phi H_{R}\right) \\
& +\alpha_{7}\left[\operatorname{Tr}\left(\tilde{\Phi} \Phi^{\dagger}+\tilde{\Phi}^{\dagger} \Phi\right) H_{R}^{\dagger} H_{R}\right]+\alpha_{8} \operatorname{Tr}\left(\Phi \Phi^{\dagger}\right) H_{R}^{\dagger} H_{R}+\beta_{1}\left(H_{L}^{\dagger} H_{L}\right)^{2}+\beta_{2}\left(H_{R}^{\dagger} H_{R}\right)^{2}+\beta_{3}\left(H_{R}^{\dagger} H_{R}\right)\left(H_{L}^{\dagger} H_{L}\right) \\
& +\gamma_{1} \operatorname{Tr}\left(\Phi^{\dagger} \Phi\right) \delta^{+} \delta^{-}+\gamma_{2} \operatorname{Tr}\left[\tilde{\Phi} \Phi^{\dagger}+\tilde{\Phi}^{\dagger} \Phi\right] \delta^{+} \delta^{-}+\gamma_{3} H_{L}^{\dagger} H_{L} \delta^{+} \delta^{-}+\gamma_{4} H_{R}^{\dagger} H_{R} \delta^{+} \delta^{-}+\gamma_{5}\left(\delta^{+} \delta^{-}\right)^{2} .
\end{aligned}
$$

This gives four $C P$-even, two $C P$-odd, and three charged Higgs boson states. Two $C P$-odd and two charged states are eaten up to give mass to the $Z_{R}$, $Z, W_{R}$, and $W$ gauge bosons, respectively. We will mainly focus our discussion on the charged Higgs sector, as that is the most important for the neutrino masses and the collider analysis which will be studied in this paper.

Minimizing the scalar potential of Eq. (12), we get four minimization conditions given as 


$$
\begin{aligned}
& 2\left(\lambda_{1}+4 \lambda_{2}+2 \lambda_{3}\right) v_{1} v_{2}^{2}+2 \lambda_{4} v_{2}^{3}+v_{1}\left(\alpha_{5} v_{L}^{2}+\alpha_{8} v_{R}^{2}+2 \lambda_{1} v_{1}^{2}-\mu_{1}^{2}\right)+2 v_{2}\left(\alpha_{4} v_{L}^{2}+\alpha_{7} v_{R}^{2}+3 \lambda_{4} v_{1}^{2}-\mu_{2}^{2}\right)+M_{2} v_{L} v_{R}=0, \\
& 2\left(\lambda_{1} v_{2}+3 \lambda_{4} v_{1}\right) v_{2}^{2}+v_{2}\left\{\left(\alpha_{3}+\alpha_{5}\right) v_{L}^{2}+\left(\alpha_{6}+\alpha_{8}\right) v_{R}^{2}+2\left(\lambda_{1}+4 \lambda_{2}+2 \lambda_{3}\right) v_{1}^{2}-\mu_{1}^{2}\right\}+M_{1} v_{L} v_{R} \\
& \quad+2 v_{1}\left(\alpha_{4} v_{L}^{2}+\alpha_{7} v_{R}^{2}+\lambda_{4} v_{1}^{2}-\mu_{2}^{2}\right)=0, \\
& \left\{4 \alpha_{4} v_{1} v_{2}+\alpha_{5} v_{1}^{2}+\left(\alpha_{3}+\alpha_{5}\right) v_{2}^{2}+2 \beta_{1} v_{L}^{2}+\beta_{3} v_{R}^{2}-\mu_{4}^{2}\right\} v_{L}+M_{2} v_{1} v_{R}+M_{1} v_{2} v_{R}=0, \\
& \left\{4 \alpha_{7} v_{1} v_{2}+\alpha_{8} v_{1}^{2}+\left(\alpha_{6}+\alpha_{8}\right) v_{2}^{2}+2 \beta_{2} v_{R}^{2}+\beta_{3} v_{L}^{2}-\mu_{3}^{2}\right\} v_{R}+M_{2} v_{1} v_{L}+M_{1} v_{2} v_{L}=0 .
\end{aligned}
$$

Using these conditions along with the scalar potential, the charged Higgs mass-squared matrix in the gauge basis $\left(\phi_{1}^{-*}, \phi_{2}^{+}, H_{R}^{+}, H_{L}^{+}, \delta^{+}\right)$is given as

$$
M_{H^{ \pm}}^{2}=\left(\begin{array}{ccccc}
M_{11} & M_{12} & \alpha_{3} v_{1} v_{L}-M_{2} v_{R} & \alpha_{6} v_{2} v_{R}+M_{1} v_{L} & -\alpha_{2} v_{L} v_{R} \\
M_{12} & M_{22} & \alpha_{3} v_{2} v_{L}+M_{1} v_{R} & \alpha_{6} v_{1} v_{R}-M_{2} v_{L} & \alpha_{1} v_{L} v_{R} \\
\alpha_{3} v_{1} v_{L}-M_{2} v_{R} & \alpha_{3} v_{2} v_{L}+M_{1} v_{R} & M_{33} & M_{1} v_{1}+M_{2} v_{2} & -\left(\alpha_{1} v_{2}+\alpha_{2} v_{1}\right) v_{R} \\
\alpha_{6} v_{2} v_{R}+M_{1} v_{L} & \alpha_{6} v_{1} v_{R}-M_{2} v_{L} & M_{1} v_{1}+M_{2} v_{2} & M_{44} & \left(\alpha_{1} v_{1}+\alpha_{2} v_{2}\right) v_{L} \\
-\alpha_{2} v_{L} v_{R} & \alpha_{1} v_{L} v_{R} & -\left(\alpha_{1} v_{2}+\alpha_{2} v_{1}\right) v_{R} & \left(\alpha_{1} v_{1}+\alpha_{2} v_{2}\right) v_{L} & M_{55}
\end{array}\right),
$$

where

$$
\begin{aligned}
& M_{11}=\left(-M_{2} v_{1} v_{L} v_{R}+M_{1} v_{2} v_{L} v_{R}+\alpha_{3} v_{1}^{2} v_{L}^{2}+\alpha_{6} v_{2}^{2} v_{R}^{2}\right) /\left(v_{1}^{2}-v_{2}^{2}\right), \\
& M_{12}=\left(M_{1} v_{1} v_{L} v_{R}-M_{2} v_{2} v_{L} v_{R}+\alpha_{3} v_{1} v_{2} v_{L}^{2}+\alpha_{6} v_{1} v_{2} v_{R}^{2}\right) /\left(v_{1}^{2}-v_{2}^{2}\right), \\
& M_{22}=\left(-M_{2} v_{1} v_{L} v_{R}+M_{1} v_{2} v_{L} v_{R}+\alpha_{3} v_{2}^{2} v_{L}^{2}+\alpha_{6} v_{1}^{2} v_{R}^{2}\right) /\left(v_{1}^{2}-v_{2}^{2}\right), \\
& M_{33}=-\frac{1}{v_{L}}\left(M_{2} v_{1} v_{R}+M_{1} v_{2} v_{R}\right)+\alpha_{3}\left(v_{2}^{2}-v_{1}^{2}\right), \\
& M_{44}=-\frac{1}{v_{R}}\left(M_{2} v_{1} v_{L}+M_{1} v_{2} v_{L}\right)+\alpha_{6}\left(v_{2}^{2}-v_{1}^{2}\right), \\
& M_{55}=\gamma_{1}\left(v_{1}^{2}+v_{2}^{2}\right)+4 \gamma_{2} v_{1} v_{2}+\gamma_{3} v_{L}^{2}+\gamma_{4} v_{R}^{2}-\mu_{5}^{2} .
\end{aligned}
$$

This $5 \times 5$ charged Higgs mass-squared matrix can be diagonalized to obtain their mass eigenvalues as

$$
M_{\text {Diag }}^{2}=V^{\dagger} M_{H^{ \pm}}^{2} V,
$$

where $M_{\text {Diag }}^{2}$ is the diagonalized charged Higgs boson mass-squared matrix and $V$ is the corresponding diagonalizing matrix. There are two zero eigenvalues corresponding to the two Goldstone bosons absorbed by the $W_{R}^{ \pm}$and $W^{ \pm}$ bosons to give them mass. The Goldstone bosons primarily consist of $H_{R}^{ \pm}$and $\phi_{1}^{ \pm}$states, as their corresponding doublet neutral fields get the large nonzero VEVs. The other three eigenstates give the three physical charged Higgses and are linear combinations of $\phi_{2}^{ \pm}, H_{L}^{ \pm}$, and $\delta^{ \pm}$. Flavor constraints, such as, $K^{0}-\bar{K}^{0}$ and $B^{0}-\bar{B}^{0}$ mixings require the neutral component of the bidoublet field $\phi_{2}^{0}$ mass to be heavier than $15 \mathrm{TeV}$ [14], forcing its charged counterpart to be very massive as well. So, $\delta^{ \pm}$can primarily mix only with $H_{L}^{ \pm}$as $\phi_{2}^{ \pm}$is effectively decoupled, owing to its large mass. We will consider two scenarios for our analysis: one in which the lightest charged Higgs consists almost entirely of the charged singlet field $\delta^{ \pm}$and another in which the lightest physical state is an almost equal admixture of $\delta^{ \pm}$and $H_{L}^{ \pm}$. In Table I, we provide four benchmark points for the lightest charged Higgs boson $H_{1}^{ \pm}$, two for the minimal mixing and two for the maximal mixing scenarios. We also cross-check the corresponding scalar and pseudoscalar neutral Higgs bosons for the set of parameters that we use to generate the above charged Higgs masses, given in Table I. We ensure that the lightest scalar Higgs boson mass is $125 \mathrm{GeV}$ and the pseudoscalar sector has two massless Goldstone bosons, required to give masses to the $Z_{R}$ and $Z$ bosons.

TABLE I. Lightest charged Higgs boson $H_{1}^{ \pm}$eigenstates. The first two points correspond to minimal mixing, while the next two are for maximal mixing.

\begin{tabular}{lc}
\hline \hline Mass & Composition \\
\hline 473.32 & $0.002 \phi_{2}^{+}+0.999 \delta^{+}$ \\
1000.7 & $0.002 \phi_{2}^{+}+0.999 \delta^{+}$ \\
432.58 & $0.03 \phi_{1}^{-*}-0.006 \phi_{2}^{+}+0.72 H_{L}^{+}+0.69 \delta^{+}$ \\
1000.9 & $0.03 \phi_{1}^{-*}-0.006 \phi_{2}^{+}+0.76 H_{L}^{+}+0.65 \delta^{+}$ \\
\hline \hline
\end{tabular}


The quark and lepton masses can be obtained from Eq. (4) as

$$
\begin{aligned}
M_{u}=Y^{q 1} v_{1}+Y^{q 2} v_{2}, & M_{d}=Y^{q 1} v_{2}+Y^{q 2} v_{1}, \\
M_{l}=Y^{l 1} v_{2}+Y^{l 2} v_{1}, & M_{\nu}^{D}=Y^{l 1} v_{1}+Y^{l 2} v_{2} .
\end{aligned}
$$

One can perform a simple rotation of the neutral bidoublet fields to obtain two new scalar fields,

$$
h_{1}^{0}=\frac{v_{1} \phi_{1}^{0}+v_{2} \phi_{2}^{0}}{\sqrt{v_{1}^{2}+v_{2}^{2}}}, \quad h_{2}^{0}=\frac{v_{2} \phi_{1}^{0}-v_{1} \phi_{2}^{0}}{\sqrt{v_{1}^{2}+v_{2}^{2}}} .
$$

In this rotated basis, only one of these new fields $\left(h_{1}^{0}\right)$ gets a nonzero VEV. This, along with a redefinition of the couplings, gives

$$
M_{u}=Y^{q} v_{1}^{\prime}, \quad M_{d}=\tilde{Y}^{q} v_{1}^{\prime}, \quad M_{l}=\tilde{Y}^{l} v_{1}^{\prime}, \quad M_{\nu}^{D}=Y^{l} v_{1}^{\prime},
$$

where $\left\langle h_{1}^{0}\right\rangle=v_{1}^{\prime}$ is the VEV in the redefined basis and

$$
\begin{aligned}
Y^{q} & =\frac{1}{v_{1}^{\prime}}\left(Y^{q 1} v_{1}+Y^{q 2} v_{2}\right), & \tilde{Y}^{q} & =\frac{1}{v_{1}^{\prime}}\left(Y^{q 1} v_{2}+Y^{q 2} v_{1}\right), \\
\tilde{Y}^{l} & =\frac{1}{v_{1}^{\prime}}\left(Y^{l 1} v_{2}+Y^{l 2} v_{1}\right), & Y^{l} & =\frac{1}{v_{1}^{\prime}}\left(Y^{l 1} v_{1}+Y^{l 2} v_{2}\right) .
\end{aligned}
$$

The $\delta^{+}$field is responsible for producing the Majorana mass terms in the neutrino mass matrix, which are given as [6]

$$
\begin{aligned}
\left(M_{\nu}^{L}\right)^{\alpha \gamma}= & \frac{1}{4 \pi^{2}} \lambda_{L}^{\prime \alpha \beta} m_{e_{\beta}} \sum_{i=1}^{3} \log \left(\frac{M_{h_{i}}^{2}}{m_{e_{\beta}}^{2}}\right) \\
& \times V_{5 i}\left[\left(Y_{l}^{\dagger}\right)^{\beta \gamma} V_{2 i}^{*}-\left(\tilde{Y}_{l}^{\dagger}\right)^{\beta \gamma} V_{1 i}^{*}\right]+\alpha \leftrightarrow \gamma, \\
\left(M_{\nu}^{R}\right)^{\alpha \gamma}= & \frac{1}{4 \pi^{2}} \lambda_{R}^{\alpha \beta} m_{e_{\beta}} \sum_{i=1}^{3} \log \left(\frac{M_{h_{i}}^{2}}{m_{e_{\beta}}^{2}}\right) \\
& \times V_{5 i}\left[\left(Y_{l}\right)^{\beta \gamma} V_{1 i}^{*}-\left(\tilde{Y}_{l}\right)^{\beta \gamma} V_{2 i}^{*}\right]+\alpha \leftrightarrow \gamma .
\end{aligned}
$$

Here, $\alpha, \beta$, and $\gamma$ each run from $1-3, V_{i j}$ corresponds to the $i j$ th element of the charged Higgs boson mixing matrix $V$ defined in Eq. (16), $M_{h_{i}}(i=1-3)$ is the mass of the charged Higgs boson eigenstates, and $m_{e_{\alpha}}$ is the charged lepton mass with $\alpha=1,2$, and 3 representing the electron, muon, and tau, respectively. The neutrino mass matrix would thus be a $6 \times 6$ matrix in the $\left(\nu_{L_{i}}, \nu_{R_{j}}\right)(i, j=1-3)$ basis given as

$$
M_{\nu}=\left[\begin{array}{cc}
M_{\nu}^{L} & M_{\nu}^{D} \\
\left(M_{\nu}^{D}\right)^{T} & M_{\nu}^{R}
\end{array}\right]
$$

where $M_{\nu}^{L}$ and $M_{\nu}^{R}$ are generated at one loop while $M_{\nu}^{D}$ is the neutrino Dirac mass term. With the seesaw approximation, the light neutrino mass matrix appears as a combination of the type-I and type-II seesaws:

$$
M_{\nu}=M_{\nu}^{L}-M_{\nu}^{D T} M_{\nu}^{R-1} M_{\nu}^{D} .
$$

The redefined coupling $\tilde{Y}_{l}$, which we have chosen to be diagonal, is entirely determined from the charged lepton masses as can be seen from Eq. (19). Similarly, $Y^{q}$ (chosen to be diagonal) and $\tilde{Y}^{q}$ can be determined from the up and down sector quark masses and CKM mixings. For the neutrino sector, we first choose $Y_{l}$ to be zero to get the light neutrino masses and mixings from $M_{\nu}^{L}$ alone. This approach does not work, as there are too few free parameters to fit the experimental neutrino data ( $\lambda_{L}^{\prime}$ is antisymmetric). We then consider the case with nonzero $Y_{l}$ while $\lambda_{L}^{\prime}$ is chosen to be zero. The neutrino mass generation thus becomes similar to the type-I seesaw mechanism, with the light neutrino masses arising entirely from $M_{D}^{\nu}$ and $M_{R}$ as,

$$
M_{\nu}=-M_{\nu}^{D T} M_{\nu}^{R-1} M_{\nu}^{D} .
$$

This gives us the correct experimentally observed masses and mixings for the light neutrino, and hence this is the approach we have chosen for the neutrino sector. ${ }^{2}$

The right-handed neutrino masses in this scenario are generated at one loop and proportional to the square of the charged lepton Yukawa coupling $\tilde{Y}_{l}$. Therefore, righthanded neutrino masses are quite small. As the other Yukawa coupling $Y_{l}$ is responsible for generating Dirac masses for the neutrinos, it is orders of magnitude smaller than $\tilde{Y}_{l}$ and hence does not have any impact on righthanded neutrino masses. We show the variation of the three right-handed neutrino masses $M_{N_{1,2,3}}$ with the Yukawa coupling $\lambda_{R}^{\prime}$ in Fig. 1 . As can be seen, for $\lambda_{R}^{\prime} \sim 0.1-1$, the lightest right-handed neutrino mass $M_{N_{1}}$ varies from 3 to $30 \mathrm{eV}$, while $M_{N_{2,3}}$ are in the sub-mega-electron-volts scale. In deriving this, we utilize Eq. (21), where we diagonalize the right-handed Majorana mass matrix $M_{\nu}^{R}$. The charged Higgs boson masses and mixings used to obtain the neutrino Majorana masses are the ones corresponding to the first benchmark point in Table I. We have also provided these charged Higgs boson masses and mixings in details in Appendix A. This is quite different from other left-right symmetric models in which the right-handed neutrino is naturally heavy as its mass is proportional to the righthanded symmetry breaking scale.

For our subsequent analysis, we choose $\lambda_{R}^{\prime} \sim \mathcal{O}(1)$. Since we use a type-I seesawlike structure for the neutrino

\footnotetext{
${ }^{2}$ Even if we keep both $Y_{l}$ and $\lambda_{L}^{\prime}$ to be nonzero, for which $M_{\nu}^{L} \neq 0$, the values of the elements of the $\lambda_{L}^{\prime}$ matrix satisfying the neutrino constraints turn out to be too small to have any observable consequences for our study.
} 


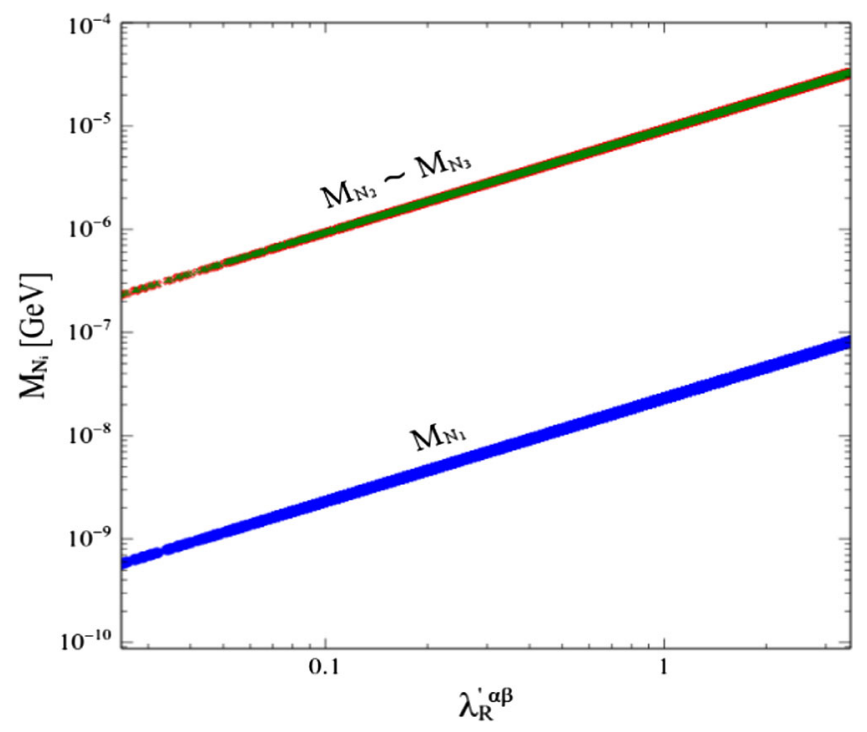

FIG. 1. Right-handed neutrino masses as a function of $\lambda_{R}^{\prime}$ for minimal charged Higgs mixing.

mass, the Dirac Yukawa couplings $Y^{l}$ in Eq. (19) are chosen accordingly to satisfy the correct neutrino oscillation parameters, given in Table II. For an illustrative example, we consider a normal hierarchy spectrum in the light neutrino sector. The allowed values for the elements of the Dirac mass matrix $M_{\nu}^{D}$ are obtained by scanning over the allowed parameter space. We have varied the elements of $\lambda_{R}^{\prime}$ matrix between 0.5 and 1 , keeping them very close to each other by allowing a spread of only $10 \%$. To generate $M_{\nu}^{R}$, we set the charged Higgs masses and mixings as given in Appendix. Figure 2 gives a scatter plot of the allowed neutrino Dirac masses (directly proportional to the Dirac Yukawa coupling $Y_{l}$ ) satisfying the experimental $3 \sigma$ ranges for the light neutrino parameters given in Table II. Here, we plot the neutrino Dirac masses along the y axis with $M_{\nu_{11}}^{D}$ along the $\mathrm{x}$ axis. This gives us an clear indication of the allowed values of the various terms in the $M_{\nu}^{D}$ matrix relative to each other. Note that the hierarchy between $\left(M_{\nu}^{D}\right)_{11},\left(M_{\nu}^{D}\right)_{12}$, and $\left(M_{\nu}^{D}\right)_{13}$ is clearly visible from Fig. 2, which shows that $\left(M_{\nu}^{D}\right)_{13}$ element of the Dirac mass matrix acquires the largest numerical values. The hierarchy

TABLE II. Experimental $3 \sigma$ ranges for light neutrino parameters. See Refs. [15,16] for further details.

$7.03 \times 10^{-5} \mathrm{eV}^{2}<\Delta m_{21}^{2}<8.09 \times 10^{-5} \mathrm{eV}^{2}$

$2.407 \times 10^{-3} \mathrm{eV}^{2}<\Delta m_{31}^{2}<2.643 \times 10^{-3} \mathrm{eV}^{2}$

$0.271<\sin ^{2} \theta_{12}<0.345$

$0.385<\sin ^{2} \theta_{23}<0.635$

$0.01934<\sin ^{2} \theta_{13}<0.02392$

$U_{P M N S}$

$\left(\begin{array}{lll}0.800 \rightarrow 0.844 & 0.515 \rightarrow 0.581 & 0.139 \rightarrow 0.155 \\ 0.229 \rightarrow 0.516 & 0.438 \rightarrow 0.699 & 0.614 \rightarrow 0.790 \\ 0.249 \rightarrow 0.528 & 0.462 \rightarrow 0.715 & 0.595 \rightarrow 0.776\end{array}\right)$ between $\left(M_{\nu}^{D}\right)_{12}$ and $\left(M_{\nu}^{D}\right)_{13}$ is larger for lower values of $\left(M_{\nu}^{D}\right)_{11}$ matrix element.

As is clear from the preceding discussion, in the present model, we have an electron-volt-scale right-handed neutrino. Hence, it may give the contribution to the relativistic degree of freedom (d.o.f) of the Universe if they equilibrate with the cosmic soup through their mixing with the active neutrinos. Recently, from Planck data, there is a strong bound on the sum of the light d.o.f., which at $2 \sigma$ gives $N_{\nu}<3.2$ and comes when we combine the $D / H$ ratio with the cosmic microwave background baryon density $[17,18]$. However, the recent LSND $[9,10]$ and MiniBooNE [11-13] data of electron excess in the antineutrino mode requires an electron-volt-scale sterile neutrino [19]. The reactor anomalies [20-23] and the gallium experiments calibration data [24-27] also hinted the presence of electron-volt-scale sterile neutrinos. Therefore, to go around the bound on the light relativistic d.o.f., a number of mechanisms have been suggested to overcome it. Among them, the popular ones are as follows. In Refs. [28-31], the authors have used secret interactions in which the sterile neutrinos are charged under some hidden symmetry mediated by the light gauge boson, resulting in the mixing between active and sterile neutrinos being suppressed due to the large thermal potential experienced by the sterile neutrinos. In Ref. [32], they have shown that relativistic d.o.f. can be alleviated if the sterile neutrino is produced in a scenario in which the reheating temperature $\left(T_{R}\right)$ is low, $T_{R}<7 \mathrm{MeV}$. The authors of Ref. [33] have shown how to reduce $N_{\nu}$ by studying the active-sterile flavor conversion. In Ref. [34], they have used mega-electron-volt dark matter to reduce $N_{\nu}$ with the help of $p$-wave annihilations. Reference [35] discusses the fact that without violating cosmology we can

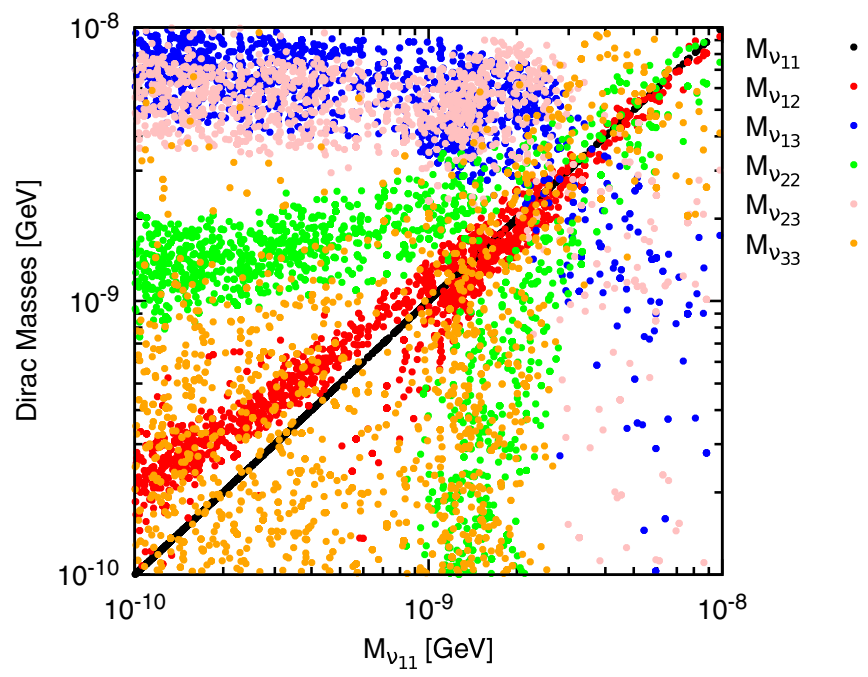

FIG. 2. Scatter plot of neutrino Dirac mass matrix elements $M_{\nu_{i j}}^{D}$ (denoted by $M_{\nu_{i j}}$ in the figure) satisfying the neutrino oscillation data in Table II. 
increase the relativistic d.o.f. by reducing the neutron-toproton ratio $(n / p)$. A number of these possible resolutions can be applied for our model. For example, we can consider the existence of secret interactions with some hidden sector particles, which would help lower the neutrino mixing between the left-handed and right-handed neutrinos. The effect of these interactions, though, would have ceased to exist at a much earlier time in the Universe, and today we would not be able to observe them anymore. Hence, our current study would not be sensitive to them. Again, the other two right-handed neutrinos are in mega-electron-volt mass range and have a warm spectrum; i.e., they are neither relativistic (which makes the problem with the cosmological structure formation [36]) nor nonrelativistic. Extensive studies in the context of structure formation for such submega-electron-volt right-handed neutrinos are there in the literature [19,37-45]. Our model can thus be made consistent with the cosmological constraints, but we have not considered them here as it is beyond the scope of this work.

\section{EXPERIMENTAL LIMITS AND COLLIDER SIGNATURE}

In Table I, we present a list of the various charged Higgs eigenstates that we consider in this study. We consider two cases with minimal mixings (thus consisting entirely of $\delta^{+}$) and two with maximal mixing of $\delta^{+}$with $H_{L}^{+}$. For these benchmark points, we study the pair production of charged Higgs states and their decay to a final state of two oppositesign charged leptons and two neutrinos. The most recent experimental bound on this process is from the ALTAS search [46] of two opposite-sign leptons and missing energy, which puts a bound of $500 \mathrm{GeV}$ if the final state is coming from pair production of two sleptons. The production cross section of the charged Higgs at the LHC is, however, much lower for our model, and even a $430 \mathrm{GeV}$ charged Higgs is safe from the LHC bounds. ${ }^{3}$ Therefore, the benchmark points we have considered are allowed by the experimental observations. The pair production of the charged Higgs at the LHC is through the $s$-channel process mediated by $\gamma, Z$, and $Z_{R}$ bosons, which gives a small production cross section. In a lepton collider, on the other hand, there is an additional $t$-channel process mediated by the neutrinos as shown in Fig. 3. Owing to the large couplings of the charged singlet with the right-handed leptons and the small masses of the right-handed neutrinos in this model, this $t$-channel process will be the major pair-production channel. The masses of the right-handed neutrinos used in our analysis are taken as

$$
M_{N_{1}}=17 \mathrm{eV}, \quad M_{N_{2}}=6.8 \mathrm{KeV}, \quad M_{N_{3}}=8.2 \mathrm{KeV},
$$

\footnotetext{
${ }^{3}$ For a set of loose cuts denoted by SF1 in Ref. [46], a production cross section for $l^{+} l^{-} E_{T}$ greater than $2 \mathrm{fb}$ is ruled out, while we only get $0.23 \mathrm{fb}$ for $M_{H^{ \pm}}=450 \mathrm{GeV}$ with similar cuts.
}
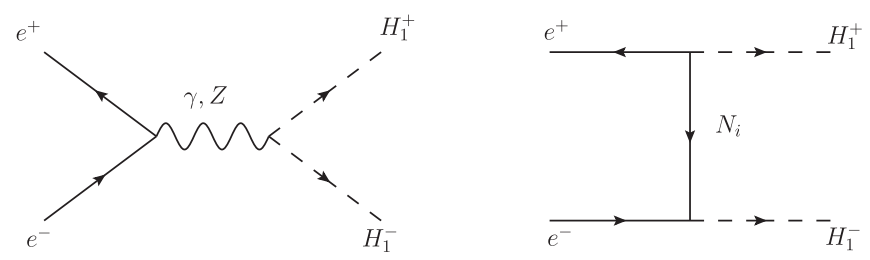

FIG. 3. Feynman diagram for the production of $H_{1}^{+} H_{1}^{-}$at $e^{+} e^{-}$ collider. The right panel diagram represents the contribution of the right-handed neutrinos in the pair-production process.

for which the values of the Yukawa coupling $\lambda_{R}^{\prime} \sim \mathcal{O}(1)$. We thus study the pair production of the charged Higgs at the $1 \mathrm{TeV}$ run of the ILC [47] and $3 \mathrm{TeV}$ run by the CLIC [48]. We include the relevant vertices in FEYnRules [49] and use MADGRAPH [50] for event generation, PYTHIA [51] for hadronization, and DELPHES [52] for detector simulation. Figure 4 shows the pair-production cross section of the charged singlet Higgs as a function of its mass for four different c.m. energies at the lepton colliders. Here, we consider the scenario of minimal mixing of the singlet charged scalar for this figure. The charged Higgs decays to a charged lepton and a right-handed neutrino and gives rise to a final state of dileptons with opposite charge $\left(l^{+}\right.$and $l^{-}$) and missing energy. Even in the case in which the charged Higgs is a mixture of $\delta^{ \pm}$and $H_{L}^{ \pm}$, this is the only kinematically allowed two-body decay channel with its branching into three-body decays being almost negligible. This is because $H_{L}$ does not couple to the quarks or leptons and its other physical states (the charged state with $H_{L}^{ \pm}$and $\delta^{ \pm}$orthogonal to the one considered here and the $C P$-odd and $C P$-even neutral states coming from $H_{L}^{0}$ ) are much heavier. Schematically, the signal looks like

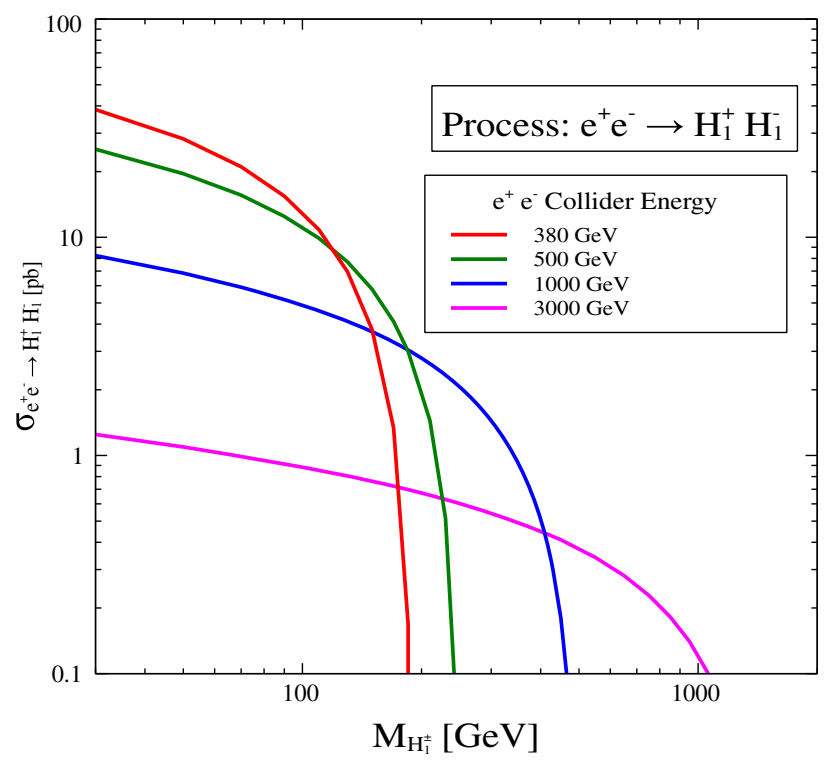

FIG. 4. Production cross section of $H_{1}^{+} H_{1}^{-}$at the $e^{+} e^{-}$collider for different center-of-mass energies. 


$$
e^{+} e^{-} \rightarrow H^{+} H^{-} \rightarrow l^{+} l^{-} E_{T}+X,
$$

where $l^{ \pm}$is either one of $e^{ \pm}, \mu^{ \pm}$, and $\tau^{ \pm}$or a combination of them. Inside the detector, the $\tau$ lepton will decay leptonically or hadronically, and a small portion of it will give an opposite-sign dilepton and will increase the signal strength. As $\tau$ decays, eventually, we get a final-state signal which consists of an opposite-sign electron $\left(e^{ \pm}\right)$or muon $\left(\mu^{ \pm}\right)$or dijet. For simulation, we consider Yukawa couplings $\lambda_{R}^{\prime}$, which are allowed by neutrino oscillation data.

Since we are interested in the opposite-sign dilepton $\left(l^{+} l^{-}\right)$and missing energy $\left(\mathbb{E}_{T}\right)$ signal in the final state, the corresponding SM dominant backgrounds are as follows:

(1) At the time of electron positron collision, the opposite-sign dilepton and missing energy can be produced as $e^{+} e^{-} \rightarrow l^{+} l^{-} Z\left(\rightarrow \nu_{l} \overline{\nu_{l}}\right)$. This includes both the $Z Z$ and the virtual photon contribution.

(2) Another dominant background is the $W^{+} W^{-}$pair production and its further leptonic decay. This can mimic the signal as $e^{+} e^{-} \rightarrow W^{+} W^{-} \rightarrow l^{+} l^{-} \nu_{l} \overline{\nu_{l}}$.

(3) Moreover, $t \bar{t}$ final state production and its subsequent decay will also affect the signal as background in the following manner: $e^{+} e^{-} \rightarrow$ $t\left(\rightarrow b l^{+} \nu_{l}\right) \bar{t}\left(\rightarrow \bar{b} l^{-} \bar{\nu}_{l}\right)$.

We do not put any veto on the light jet in our analysis. Additionally, this is to note that the signal does not comprise any $b$ jet. Therefore, a $b$ veto will reduce the backgrounds, such as $t \bar{t}$ production. Depending on the various kinematical variables, there is a clear distinction between the signal and the backgrounds as can be seen clearly in Fig. 5. The leftmost plot in Fig. 5 shows the distribution of the transverse momentum of the hardest lepton $\left(p_{T}^{l_{1}}\right)$, the one in the middle is its pseudorapidity distribution $\left(\eta_{l_{1}}\right)$, and the rightmost plot shows the missing energy distribution of the signal and background events. Following these, we can select appropriate cuts on different kinematical variables to minimize the background, while protecting the signal as much as possible. The details of the cuts, which we use in our analysis, are as follows:

(A0) We consider a signal in which final state contains two opposite-sign dileptons with missing energy, i.e., $l^{+} l^{-} \boldsymbol{E}_{T}$. We implement a minimum cut on the $p_{T}$ of the leptons, which is $p_{T, l}^{\min } \geq 10 \mathrm{GeV}$. We also implement an upper limit on the pseudorapidity, which is $\left|\eta^{l}\right|<2.5$. These cuts have been implemented at the time of generating the partonic event samples.

(A1) We select our events which contain two oppositesign dilepton.

(A2) From the left panel of Fig. 5, one can see that if we use the cut on the hardest lepton around $130 \mathrm{GeV}$ then background can be reduced. We therefore use the cut on the $p_{T}$ of the hardest lepton, which is equal to or greater than $130 \mathrm{GeV}, p_{T}^{l_{1}} \geq 130 \mathrm{GeV}$, and a relatively softer cut on the second lepton, which is $p_{T}^{l_{2}} \geq 60 \mathrm{GeV}$.

(A3) The background from $Z Z$ pair production can be safely removed by applying a $Z$ veto. We put a small window on the dilepton invariant mass $\left(m_{l l}\right)$ which is $\left|m_{l l}-91.2\right| \leq 10 \mathrm{GeV}$, and reject the events where $m_{l l}$ falls within this window.

(A4) One of the backgrounds $(t \bar{t})$ contains $b$ jets in the final state. However, the signal of our interest does not have any $b$ jets. Therefore, we have used the $b$ veto in the final state to reduce the background without affecting the signal.

(A5) From the middle panel of Fig. 5, it is evident that signal and backgrounds peak at different values of the pseudorapidity of the leading lepton. We use a tighter cut on $\eta^{l_{1}}$. We reject events which have $\left|\eta^{l_{1}}\right| \geq 1$.

(A6) The right-handed neutrinos in our scenario are very light, as they have $\sim$ electron-volt-scale to mega-electron-volt-scale masses. The decay of
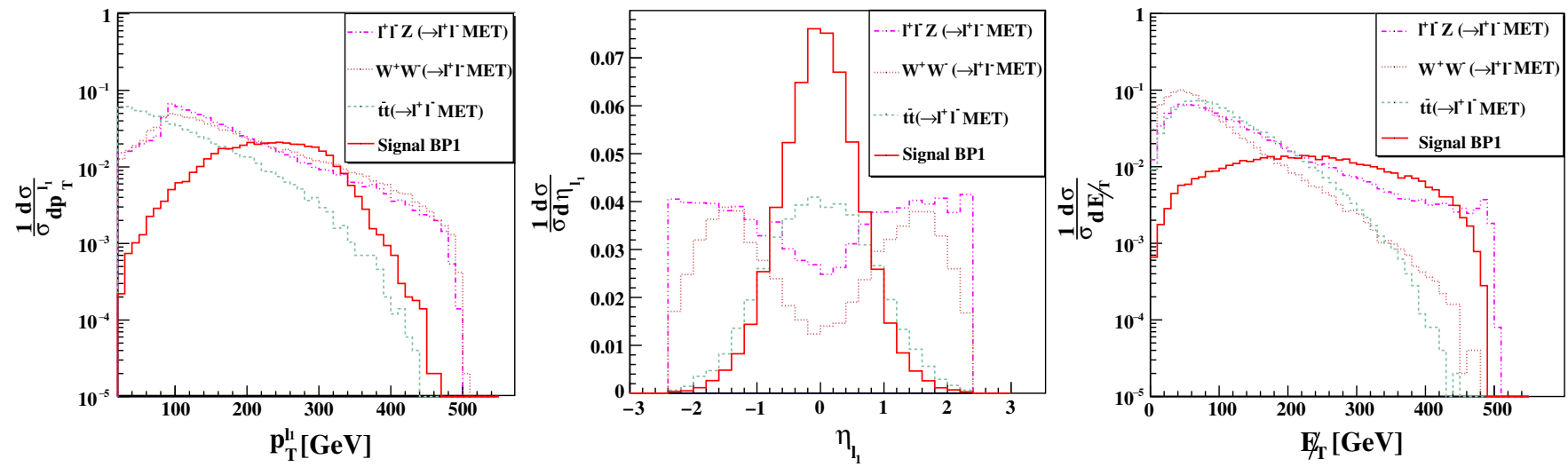

FIG. 5. Distribution of signal events and background processes for different kinematical variables. The plot on the left is the transverse momentum of the hardest lepton, the middle plot is for the pseudorapidity of the hardest lepton, and the right plot is the missing energy distribution. 
TABLE III. Cut-flow table for the obtained cross sections corresponding to the different SM backgrounds. See the text for the details of the cuts A0-A6. The c.m. energy is $\sqrt{s}=1 \mathrm{TeV}$, relevant for the ILC.

\begin{tabular}{|c|c|c|c|c|c|c|c|}
\hline \multicolumn{2}{|c|}{ SM backgrounds at $1 \mathrm{TeV}$ ILC } & \multicolumn{6}{|c|}{ Effective cross section after applying cuts (fb) } \\
\hline Channels & Cross section $(\mathrm{fb})$ & $\mathrm{A} 0+\mathrm{A} 1$ & A2 & A3 & A4 & A5 & A6 \\
\hline$l^{+} l^{-} Z\left(\rightarrow \nu_{l} \overline{\nu_{l}}\right)$ & 18.68 & 10.79 & 5.99 & 5.54 & 5.54 & 2.30 & 1.67 \\
\hline$W^{+}\left(\rightarrow l^{+} \nu_{l}\right) W^{-}\left(\rightarrow l^{-} \overline{\nu_{l}}\right)$ & 126.88 & 52.72 & 32.15 & 32.15 & 32.15 & 12.44 & 7.05 \\
\hline$t\left(\rightarrow b l^{+} \nu_{l}\right) \bar{t}\left(\rightarrow \bar{b} l^{-} \bar{\nu}_{l}\right)$ & 13.96 & 3.10 & 0.78 & 0.78 & 0.1 & 0.08 & 0.05 \\
\hline Total backgrounds & & & & & & & 8.77 \\
\hline
\end{tabular}

TABLE IV. Cut-flow table for the obtained cross sections corresponding to the SM backgrounds. The details of the cuts A0-A6 are mentioned in the text. We perform the simulation for the $3 \mathrm{TeV}$ CLIC.

\begin{tabular}{|c|c|c|c|c|c|c|c|}
\hline \multicolumn{2}{|c|}{ SM backgrounds at $3 \mathrm{TeV}$ CLIC } & \multicolumn{6}{|c|}{ Effective cross section after applying cuts (fb) } \\
\hline Channels & Cross section $(\mathrm{fb})$ & $\mathrm{A} 0+\mathrm{A} 1$ & A2 & A3 & A4 & A5 & A6 \\
\hline$l^{+} l^{-} Z\left(\rightarrow \nu_{l} \overline{\nu_{l}}\right)$ & 6.33 & 3.0 & 2.89 & 2.86 & 2.86 & 0.54 & 0.44 \\
\hline$W^{+}\left(\rightarrow l^{+} \nu_{l}\right) W^{-}\left(\rightarrow l^{-} \bar{\nu}_{l}\right)$ & 13.85 & 5.45 & 5.1 & 5.1 & 5.1 & 1.34 & 1.13 \\
\hline$t\left(\rightarrow b l^{+} \nu_{l}\right) \bar{t}\left(\rightarrow \bar{b} l^{-} \bar{\nu}_{l}\right)$ & 1.76 & 0.05 & 0.02 & 0.02 & 0.005 & 0.002 & 0.002 \\
\hline Total backgrounds & & & & & & & 1.57 \\
\hline
\end{tabular}

right-handed neutrinos cannot happen inside the detector. Hence, they will be undetected and will give missing energy. We show the distribution of $\mathbb{E}_{T}$ in the right panel of Fig. 5. To reduce the background, we also use a cut on the missing energy, which is $\mathbb{E}_{T}>80 \mathrm{GeV}$. This further enhances the signal-to-background ratio.

Using the above-mentioned cuts, we can reduce the background significantly while keeping the signal at a significant level. In Tables III and IV, we show the background cross sections at $1 \mathrm{TeV}$ ILC and $3 \mathrm{TeV}$ CLIC experiments, respectively, after implementing all the abovementioned cuts. The dominant background is $W^{ \pm} W^{\mp}$ production, which has a cross section $126.88 \mathrm{fb}$ (for the $1 \mathrm{TeV}$ ILC) at the partonic level. It is evident that the backgrounds become quite small $\sigma \sim 7,1$ fb for the ILC and CLIC, respectively, after the cuts.

The signal cross sections and their statistical significance over the background are given in Table $\mathrm{V}$ for the chosen benchmark points. Clearly, the case with no mixing in the Higgs state gives a much larger cross section. This is because the $\delta^{ \pm} l_{R}^{\mp} \nu_{R}$ vertex is primarily responsible for the charged Higgs pair production. The mixing of $\delta^{ \pm}$with $H_{L}^{ \pm}$ will only introduce an extra factor of $\cos ^{4} \theta$ in the pairproduction cross section, in which $\theta$ is the mixing angle, resulting in a decrease in the cross section. As is evident, the cross section is enormous in the lepton collider. As an illustrative example, for a $1 \mathrm{TeV}$ charged Higgs $H_{1}^{ \pm}$, the partonic cross section is $\sigma \sim 100 \mathrm{fb}$. After the cuts, the cross section reduces to $\sigma \sim 27 \mathrm{fb}$. This is an order of magnitude larger than the after-cut background cross section. We compute the statistical significance $(\mathcal{S})$ of the signal over the background using the following expression:

$$
\mathcal{S}=\sqrt{2 \times\left[(s+b) \ln \left(1+\frac{s}{b}\right)-s\right]} .
$$

In the above, $s$ and $b$ denote the signal and background events. The significance is shown in Table V. As expected, the case with zero mixing has a much better significance of the signal over the background, boosting its chances to be discovered even in the early run of the upcoming lepton

TABLE V. Cut-flow table of signal cross section at the *1 TeV ILC and 3 TeV CLIC after applying the different cuts. We also show the statistical significance over the background.

\begin{tabular}{|c|c|c|c|c|c|c|c|c|c|c|c|c|}
\hline \multicolumn{5}{|c|}{ Signal at $e^{+} e^{-}$collider } & \multicolumn{6}{|c|}{ Effective cross section after cuts (fb) } & \multicolumn{2}{|c|}{ Statistical significance $(\mathcal{S})$} \\
\hline & C.M. energy (TeV) & Mass $(\mathrm{GeV})$ & Mixing & $\mathrm{CS}(\mathrm{fb})$ & $\mathrm{A} 0+\mathrm{A} 1$ & A2 & A3 & A4 & A5 & A6 & $\mathcal{L}=1 \mathrm{fb}^{-1}$ & $\mathcal{L}=3 \mathrm{fb}^{-1}$ \\
\hline $\mathrm{BP} 1$ & 1 & 473.32 & Zero & 192.67 & 79.75 & 62.13 & 62.02 & 62.02 & 57.78 & 53.63 & 11.73 & 20.32 \\
\hline $\mathrm{BP} 2$ & 3 & 1000.70 & Zero & 100.31 & 38.21 & 35.57 & 35.56 & 35.55 & 28.08 & 27.07 & 10.78 & 18.67 \\
\hline BP3 & 1 & 432.58 & Half & 49.50 & 19.19 & 14.62 & 14.59 & 14.59 & 13.54 & 12.51 & 3.56 & 6.174 \\
\hline BP4 & 3 & 1000.92 & Half & 17.86 & 6.83 & 6.33 & 6.33 & 6.33 & 5.08 & 4.99 & 2.96 & 5.13 \\
\hline
\end{tabular}


colliders. In particular, we show that only $\mathcal{L}=1 \mathrm{fb}^{-1}$ luminosity is required in the zero-mixing scenario to discover charged Higgs $H_{1}^{ \pm}$with mass range $473 \mathrm{GeV}-$ $1 \mathrm{TeV}$. For the relatively less optimistic scenario of halfmixing, $3 \mathrm{fb}^{-1}$ will be required to claim discovery.

\section{CONCLUSION}

In this work, we have studied left-right symmetric extension of the Zee model. The model has very different characteristic features as compared to the minimal left-right symmetric model. It is well known that the basic Zee model is ruled out from light neutrino mass and mixing constraints. Going to the left-right symmetric framework, it is possible to evade the tension with the neutrino oscillation data. The model consists of three lighter right-handed neutrino states, which can have masses from mega-electron-volt down to electron-volt scale. Additionally, the model also contains an additional charged scalar $\delta^{ \pm}$. The charged scalar, due to its additional interaction with charged leptons and right-handed neutrinos, can be copiously produced at a lepton collider via the $t$-channel processes.

We discuss light neutrino mass generation in this model and fit the observed data. The light neutrino mass matrix is a combination of both the type-I and type-II seesaw matrices. The type-II contribution and the right-handed neutrino mass matrix, which participates in the type-I seesaw, however, are generated through the one-loop process with the charged leptons and charged Higgs fields as mediators. We fit the observed light neutrino mass square differences and the Pontecorvo-Maki-Nakagawa-Sakata (PMNS) mixing in this model and derive constraints on model parameters. With the set of parameters, which satisfy the neutrino mass constraints, we extensively analyze the charged Higgs phenomenology at the $1 \mathrm{TeV}$ ILC and $3 \mathrm{TeV}$ CLIC. Owing to the extra interaction of the charged Higgs with the right-handed neutrinos and for moderately large Yukawa couplings, the cross section at an $e^{+} e^{-}$collider is enormous, as compared to the LHC. We find that in the most optimistic scenario, in which the lighter charged Higgs state $H_{1}^{ \pm}$is a pure charged scalar state $\delta^{ \pm}$, the cross section for pair production of the charged Higgs can be $\sigma \sim \mathcal{O}(1) \mathrm{pb}$ for $M_{H^{ \pm}} \sim 473 \mathrm{GeV}$, and c.m. energy $\sqrt{s}=1 \mathrm{TeV}$. For the CLIC, which can operate with c.m. energy $\sqrt{s}=3 \mathrm{TeV}$, the charged Higgs of mass $1 \mathrm{TeV}$ is also accessible $(\sigma \sim 100 \mathrm{fb}$ for pair production).
We consider the subsequent decay of the charged Higgs into a lepton and a neutrino, which is the only possible channel for this model. This leads to the final states $l^{+} l^{-}+\mathscr{E}_{T}$, which we analyze in detail, taking into account detector simulation. We show that a discovery of the charged Higgs of mass in between 473 and $1000 \mathrm{GeV}$ in the dilepton $+\mathbb{E}_{T}$ will require only $1-3 \mathrm{fb}^{-1}$ integrated luminosity at an $e^{+} e^{-}$collider, operating with c.m. energy $\sqrt{s}=1,3 \mathrm{TeV}$. Therefore, this model can most economically be tested at the very early run of the ILC or CLIC.

\section{ACKNOWLEDGMENTS}

M. M. would like to acknowledge DST-INSPIRE research Grant No. IFA14-PH-99 and the hospitality of CHEP, IISc, Bengaluru, where part of the discussion has been carried out. A. P. is supported by the SERB National Postdoctoral Fellowship (Grant No. PDF/2016/000202). S. K. thanks Professor Sandhya Choubey for discussions. S. $\mathrm{K}$. also acknowledges the cluster computing facility at Harish-Chandra Research Institute (http://cluster.hri.res.in). He would also like to thank the Department of Atomic Energy (DAE) Neutrino Project of Harish-Chandra Research Institute. The authors would like to thank Doctor Arnab Dasgupta for very useful discussions at the early stage of this work.

\section{APPENDIX: CHARGED HIGGS BOSON EIGENSTATES USED FOR NEUTRINO PHENOMENOLOGY}

Here, we list the charged Higgs boson masses and mixings that have been used for the neutrino phenomenology in our study. We consider that the lightest charged Higgs boson $H_{1}^{ \pm}$has a mass around $473 \mathrm{GeV}$ and is almost entirely consisting of the singlet charged Higgs field $\delta^{ \pm}$. The charged Higgs boson states, after diagonalization, consist of two Goldstone bosons, $G_{1}^{ \pm}$and $G_{2}^{ \pm}$, and three physical charged Higgs bosons with

$$
\begin{gathered}
M_{H_{1}^{ \pm}}=473.32 \mathrm{GeV}, \quad M_{H_{2}^{ \pm}}=2534.94 \mathrm{GeV}, \\
M_{H_{3}^{ \pm}}=15.95 \mathrm{TeV} .
\end{gathered}
$$

The corresponding eigenstates can be identified as

$$
\left(\begin{array}{c}
H_{1}^{ \pm} \\
H_{2}^{ \pm} \\
H_{3}^{ \pm} \\
G_{1}^{ \pm} \\
G_{2}^{ \pm}
\end{array}\right)=\left(\begin{array}{ccccc}
0.0000127106 & 0.00225768 & 0.000109819 & 0.0000433475 & 0.999997 \\
-0.114973 & 0.000916758 & -0.993368 & 0.0000176018 & 0.000108482 \\
-0.000105399 & -0.999813 & -0.000910601 & -0.0191964 & 0.0022582 \\
-0.00574986 & -0.0191961 & 0.000665494 & 0.999799 & 4.70382 \times 10^{-16} \\
0.993352 & -0.00011112 & -0.114971 & 0.00578718 & 2.76194 \times 10^{-18}
\end{array}\right)\left(\begin{array}{l}
\phi_{1}^{ \pm} \\
\phi_{2}^{ \pm} \\
H_{L}^{ \pm} \\
H_{R}^{ \pm} \\
\delta^{ \pm}
\end{array}\right)
$$

The $5 \times 5$ matrix in the above is the charged Higgs boson rotation matrix $V$. 
[1] P. Minkowski, Phys. Lett. 67B, 421 (1977); T. Yanagida, Proceedings of the Workshop on Unified Theories and Baryon Number in the Universe, Tsukuba, 1979, edited by A. Sawada and A. Sugamoto (KEK, Tsukuba, Japan, 1979), Report No. 79-18, http://inspirehep.net/record/19833; S. Glashow, in Quarks and Leptons, Cargèse 1979, edited by M. Lévy et al. (Plenum, New York, 1980); M. GellMann, P. Ramond, and R. Slansky, Proceedings of the Supergravity Stony Brook Workshop, New York, 1979, edited by P. Van Niewenhuizen and D. Freeman (NorthHolland, Amsterdam, 1979); R. Mohapatra and G. Senjanović, Phys. Rev. Lett. 44, 912 (1980).

[2] E. Ma, Phys. Rev. Lett. 81, 1171 (1998); F. Bonnet, M. Hirsch, T. Ota, and W. Winter, J. High Energy Phys. 07 (2012) 153; D. A. Sierra, A. Degee, L. Dorame, and M. Hirsch, J. High Energy Phys. 03 (2015) 040.

[3] A. Zee, Phys. Lett. 93B, 389 (1980); 95B, 461(E) (1980).

[4] P. H. Frampton, M. C. Oh, and T. Yoshikawa, Phys. Rev. D 65, 073014 (2002); Y. Koide, Nucl. Phys. B, Proc. Suppl. 111, 294 (2002); X. G. He, Eur. Phys. J. C 34, 371 (2004); J. Herrero-Garca, T. Ohlsson, S. Riad, and J. Wirn, J. High Energy Phys. 04 (2017) 130.

[5] R. N. Mohapatra and J. C. Pati, Phys. Rev. D 11, 566 (1975); G. Senjanovic and R. N. Mohapatra, Phys. Rev. D 12, 1502 (1975).

[6] P. F. Perez, C. Murgui, and S. Ohmer, Phys. Rev. D 94, 051701 (2016).

[7] P. F. Perez and C. Murgui, Phys. Rev. D 95, 075010 (2017).

[8] M. A. B. Beg and H.-S. Tsao, Phys. Rev. Lett. 41, 278 (1978); R. N. Mohapatra and G. Senjanovic, Phys. Lett. 79B, 283 (1978); K. S. Babu and R. N. Mohapatra, Phys. Rev. D 41, 1286 (1990); S. M. Barr, D. Chang, and G. Senjanovic, Phys. Rev. Lett. 67, 2765 (1991); R. N. Mohapatra and A. Rasin, Phys. Rev. Lett. 76, 3490 (1996); 76, 3490 (1996); 79, 4744 (1997); Phys. Rev. D 65, 016005 (2001); 82, 116008 (2010).

[9] C. Athanassopoulos et al. (LSND Collaboration), Phys. Rev. Lett. 75, 2650 (1995).

[10] A. Aguilar-Arevalo et al. (LSND Collaboration), Phys. Rev. D 64, 112007 (2001).

[11] A. A. Aguilar-Arevalo et al. (MiniBooNE Collaboration), Phys. Rev. Lett. 98, 231801 (2007).

[12] A. A. Aguilar-Arevalo et al. (MiniBooNE Collaboration), Phys. Rev. Lett. 105, 181801 (2010).

[13] A. A. Aguilar-Arevalo et al. (MiniBooNE Collaboration), Phys. Rev. Lett. 110, 161801 (2013).

[14] G. Ecker, W. Grimus, and H. Neufeld, Phys. Lett. 127B, 365 (1983); 132B, 467 (1983); R. N. Mohapatra, G. Senjanovic, and M. D. Tran, Phys. Rev. D 28, 546 (1983); M.E. Pospelov, Phys. Rev. D 56, 259 (1997); Y. Zhang, H. An, X. Ji, and R. N. Mohapatra, Nucl. Phys. B802, 247 (2008); A. Maiezza, M. Nemevsek, F. Nesti, and G. Senjanovic, Phys. Rev. D 82, 055022 (2010); for bounds on $W_{R}$ mass from radiative correction effects, see $\mathrm{J}$. Chakrabortty, J. Gluza, R. Sevillano, and R. Szafron, J. High Energy Phys. 07 (2012) 038.

[15] F. Capozzi, E. Di Valentino, E. Lisi, A. Marrone, A. Melchiorri, and A. Palazzo, Phys. Rev. D 95, 096014 (2017).
[16] I. Esteban, M. C. Gonzalez-Garcia, M. Maltoni, I. MartinezSoler, and T. Schwetz, J. High Energy Phys. 01 (2017) 087; , NuFIT 3.2, 2018, www.nu-fit.org.

[17] P. A. R. Ade et al. (Planck Collaboration), Astron. Astrophys. 594, A13 (2016).

[18] R. H. Cyburt, B. D. Fields, K. A. Olive, and T. H. Yeh, Rev. Mod. Phys. 88, 015004 (2016).

[19] K. N. Abazajian et al., arXiv:1204.5379.

[20] G. Mention, M. Fechner, T. Lasserre, T. A. Mueller, D. Lhuillier, M. Cribier, and A. Letourneau, Phys. Rev. D 83, 073006 (2011).

[21] T. A. Mueller et al., Phys. Rev. C 83, 054615 (2011).

[22] P. Huber, Phys. Rev. C 84, 024617 (2011); 85, 029901 (2012).

[23] J. N. Abdurashitov et al., Phys. Rev. C 73, 045805 (2006).

[24] J. N. Bahcall, P. I. Krastev, and E. Lisi, Phys. Lett. B 348, 121 (1995).

[25] C. Giunti and M. Laveder, Mod. Phys. Lett. A 22, 2499 (2007).

[26] C. Giunti and M. Laveder, Phys. Rev. D 82, 053005 (2010).

[27] C. Giunti and M. Laveder, Phys. Rev. C 83, 065504 (2011).

[28] B. Dasgupta and J. Kopp, Phys. Rev. Lett. 112, 031803 (2014).

[29] S. Hannestad, R. S. Hansen, and T. Tram, Phys. Rev. Lett. 112, 031802 (2014).

[30] J. F. Cherry, A. Friedland, and I. M. Shoemaker, arXiv:1605 .06506 .

[31] X. Chu, B. Dasgupta, M. Dentler, J. Kopp, and N. Saviano, J. Cosmol. Astropart. Phys. 11 (2018) 049.

[32] C. E. Yaguna, J. High Energy Phys. 06 (2007) 002.

[33] N. Saviano, A. Mirizzi, O. Pisanti, P. D. Serpico, G. Mangano, and G. Miele, Phys. Rev. D 87, 073006 (2013).

[34] C. M. Ho and R. J. Scherrer, Phys. Rev. D 87, 065016 (2013).

[35] M. Giovannini, H. Kurki-Suonio, and E. Sihvola, Phys. Rev. D 66, 043504 (2002).

[36] K. Abazajian, E. R. Switzer, S. Dodelson, K. Heitmann, and S. Habib, Phys. Rev. D 71, 043507 (2005); R. de Putter, O. Mena, E. Giusarma, S. Ho, A. Cuesta et al., Astrophys. J. 761, 12 (2012).

[37] P. Bode, J. P. Ostriker, and N. Turok, Astrophys. J. 556, 93 (2001).

[38] S. H. Hansen, J. Lesgourgues, S. Pastor, and J. Silk, Mon. Not. R. Astron. Soc. 333, 544 (2002).

[39] A. Boyarsky, J. Lesgourgues, O. Ruchayskiy, and M. Viel, J. Cosmol. Astropart. Phys. 05 (2009) 012.

[40] M. R. Lovell, V. Eke, C. S. Frenk, L. Gao, A. Jenkins, T. Theuns, J. Wang, S. D. M. White, A. Boyarsky, and O. Ruchayskiy, Mon. Not. R. Astron. Soc. 420, 2318 (2012).

[41] D. Boyanovsky and J. Wu, Phys. Rev. D 83, 043524 (2011).

[42] D. Boyanovsky, Phys. Rev. D 83, 103504 (2011).

[43] F. Villaescusa-Navarro and N. Dalal, J. Cosmol. Astropart. Phys. 03 (2011) 024.

[44] A. Merle and V. Niro, Phys. Rev. D 88, 113004 (2013).

[45] M. Drewes et al., J. Cosmol. Astropart. Phys. 01 (2017) 025.

[46] M. Aaboud et al. (ATLAS Collaboration), Eur. Phys. J. C 78, 995 (2018).

[47] H. Baer et al., arXiv:1306.6352; C. Adolphsen et al., arXiv: 1306.6353; arXiv:1306.6328; T. Behnke et al., arXiv:1306 .6329; arXiv:1306.6327.

[48] H. Abramowicz et al. (CLIC Detector and Physics Study Collaboration), arXiv:1307.5288; D. Dannheim et al., 
arXiv:1208.1402; E. Accomando et al. (CLIC Physics Working Group), arXiv:hep-ph/0412251; L. Linssen et al., arXiv:1202.5940; N. A. Tehrani, J.-J. Blaising, B. Cure, D. Dannheim, F. D. Ramos, K. Elsener et al., CLICdet: The post-CDR CLIC detector model, CLIC, Report No. CLICdpNote-2017-001, 2017, http://inspirehep.net/record/19833.

[49] A. Alloul, N. D. Christensen, C. Degrande, C. Duhr, and B. Fuks, Comput. Phys. Commun. 185, 2250 (2014).

[50] J. Alwall, R. Frederix, S. Frixione, V. Hirschi, F. Maltoni, O. Mattelaer, H.-S. Shao, T. Stelzer, P. Torrielli, and M. Zaro,
J. High Energy Phys. 07 (2014) 079; J. Alwall, M. Herquet, F. Maltoni, O. Mattelaer, and T. Stelzer, J. High Energy Phys. 06 (2011) 128.

[51] T. Sjostrand, S. Mrenna, and P. Z. Skands, J. High Energy Phys. 05 (2006) 026.

[52] J. de Favereau, C. Delaere, P. Demin, A. Giammanco, V. Lemaître, A. Mertens, and M. Selvaggi (DELPHES 3 Collaboration), J. High Energy Phys. 02 (2014) 057; M. Selvaggi, J. Phys. Conf. Ser. 523, 012033 (2014); A. Mertens, J. Phys. Conf. Ser. 608, 012045 (2015). 